\title{
Design and Development of Bionic Eye for Visually Challenged People using Raspberry Pi Processor
}

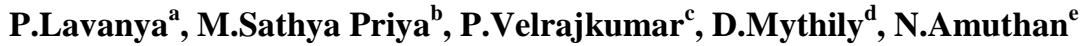 \\ ${ }^{a}$ ECE Department, M.N.M Jain Engineering College, Chennai \\ ${ }^{\mathbf{b}, \mathbf{d}}$ ECE Department, TJS Engineering College, Chennai \\ ${ }^{c}$ EEE Department, CMR Institute of Technology, Bengaluru \\ ${ }^{\mathrm{e}}$ EEE Department, AMC Engineering College, Bengaluru \\ a plavanyame@ gmail.com, ${ }^{\mathrm{b}}$ sathya77.ashok@ gmail.com, ${ }^{\mathrm{c}}$ velrajkumar.p@ cmrit.ac.in, ${ }^{\mathrm{d}}$ jemimah2007@gmail.com, ${ }^{\mathrm{e}}$ \\ amuthannadar@gmail.com
}

Article History: Received: 10 November 2020; Revised 12 January 2021 Accepted: 27 January 2021; Published online: 5 April 2021

\begin{abstract}
Bionic Eye will play a major role in the future development of visually challenged people. This research focuses on design and development of Bionic Eye to detect the hurdles for visually challenged people. This intelligence system uses the shape and movement of an object for detection and tracking. The object recognition rate is improved with the help of Stochastic Decent Gradient algorithm. Raspberry Pi is the processor used for the Bionic Eye as it gives out command on the object detection and the data from the camera is collected and then transmitted to the system. The distance and object movement is obtained by ultrasonic sensor. The range of the ultrasonic sensor is set and the distance is measured. A camera is used for capturing the object. A voice output saying "there is an object in front of you" is heard after an object is detected. The accuracy of the object detection is obtained by the deep learning algorithm. Increasing the recognition rate is the main advantage over object detection systems.
\end{abstract}

Keywords: Raspberry Pi, Bionic Eye, Stochastic Decent Gradient Algorithm, Camera, Ultrasonic sensor.

\section{Introduction}

Visually challenged people in the world are around 290 million, where 40 million people have no vision; 250 million people have less vision. Most of them are above 50 years. The Bionic Eye - a visual aid for the impaired people is an object detection device. This model uses deep learning algorithm in order to detect objects at high data accuracy rate. It also uses an ultrasonic sensor for measuring the distance and a voice note is received from the audio jack. This paper gives an overview of object detection technique to channelize the objects through open computer vision and deep learning.

Object detection is a computer technology for computer vision and image processing that deals with the detection, in digital images and videos, of instances of semantic objects of a certain class (such as people, houses, or cars). In many fields of computer vision, object recognition has applications, including image processing and video surveillance. Algorithms for object detection usually use machine learning or deep learning to generate useful results. The design network architecture is needed to learn the features for the objects of interest in order to train a custom object detector from scratch. Using deep learning leverage transfer learning, many object detection workflows use an approach that allows you to start with a pre-trained network and then fine-tune it for the specific application.

This proposed work is an attempt to design an object detection module for visually impaired people which is the Bionic Eye - a visual aid system. It uses deep learning algorithms for auto detection of objects. This deals with the design and development of a system for the aid of visually challenged to roam outside world. The prototype is a model based on object detection. It can be used as a wearable like belt, glass, hat etc. This prototype uses Raspberry $\mathrm{Pi}$ as the processor with a memory of $16 \mathrm{~GB}$. An ultrasonic sensor is interfaced with the processor to detect the distance of the obstacle.

\section{Review of Object Detection}

Andreas H. Pech et al[1] outlines a new approach to Ultrasonic Signal Analysis for pedestrian detection in vehicles where the ultrasonic sensor tests signals dispersed back from the obstacle by task-specific methods of signal analysis. Charmi T. Patel et al[2] developed multi sensor-based object detection in an indoor environment for visually impaired people. The device consists of a multi sensor-based system for object detection using statistical parameters on a captured image, which is further validated using the algorithm of the support vector 
machine. Deepthi Jain B et al[3] suggested Image Recognition Visual Assistance for Blinds, a visual help device in which the user accepts speech commands. Its functionality deals with the recognition of artifacts and signboards that use Raspberry Pi as the Open CV interface processor.Hot Glass Human Face, Object and Textual Recognition for Visually Disabled was presented by Diwakar Srinath A et al[4], and the device was proposed to practically render visually impaired people present. Sumeet Sanjay Walam et al[5] presented the Raspberry Pi Object Detection and Separation document, which is used to detect and distinguish the object by color from the collection. A light intensity camera to frequency converter system performs the detection of the relevant color. Sushrut Nagesh Kulkarni et al[6] proposed Automated System Object Sorting using Raspberry Pi. Here, an image is scanned first, and is then analyzed to detect the object's shape and color using Open CV. Rahul Kumar et al[7] developed A Novel Visually Impaired Approach using Object Recognition, a device that detects the object prior to the recursive neural network using the neural network and also produces an audio output and recognizes the color of the object in the second module using HSI color space to help visually impaired people detect the object and its color in front of them. Vivek Kishore Bhanseet al [8] proposed Face Detection and Tracking using Image Processing on Raspberry Pi, the kit uses Raspberry Pi as the processor control and a web camera to process and detect the image using Open CV, which then tracks and recognizes the human face. The Stochastic Gradient Decent Algorithm and Raspberry Pi are proposed to control and tracking the robotic movement [9-12].

\section{Materials and Methods}

Object detection system block diagram is shown in fig.1. Obstacle distance is detected by processor interfaced ultrasonic sensor. Nearly 1000 datasets for 5-10 objects are collected and the classes are trained. Using machine learning algorithm, the test images are programmed using the python language to increase the detection rate. These trained objects increase the detection rate. The distance is then notified by the audio output using the text to speech convertor.

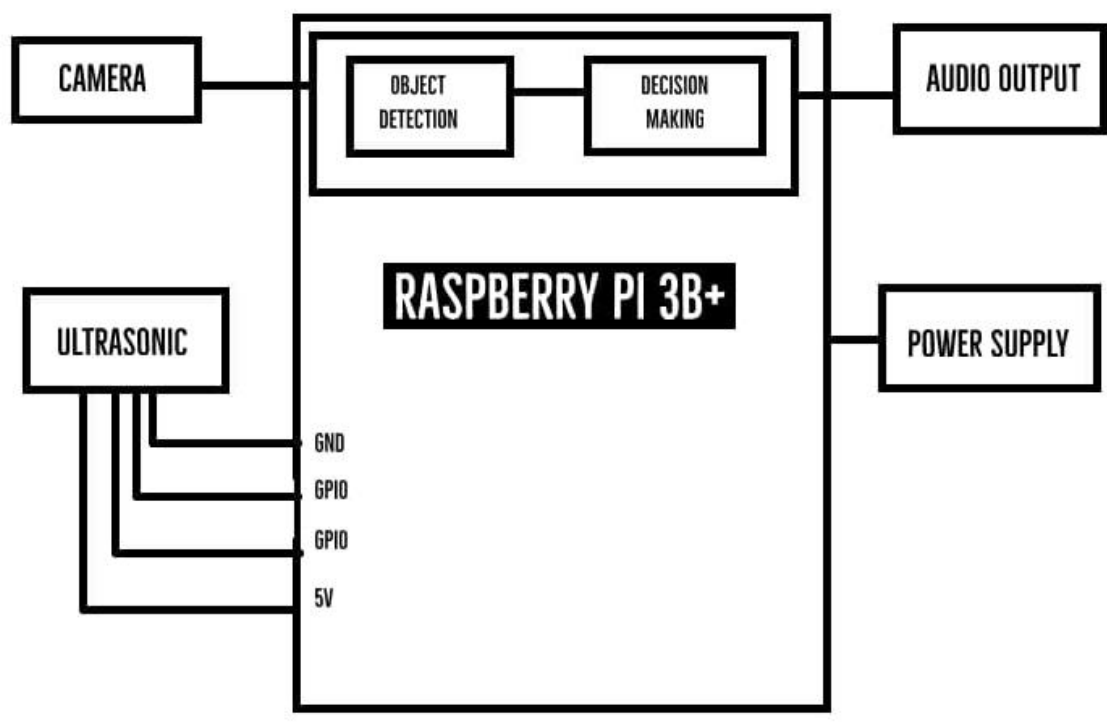

Figure 1 System Block Diagram

Updated version of Rasberry Pi is Raspberry Pi 3 Model B+ which is shown in fig. 2 and is the latest product in the Raspberry Pi 3 whose bandwidth $2.4 \mathrm{GHz}$ and $5 \mathrm{GHz}$ W LAN, faster Ethernet, and Power over Ethernet capability via a separate Power over Ethernet HAT. BCM2837 SoC (System on Chip) with a 1.4 GHz 64-bit quad-core A-53 processor, with $512 \mathrm{~KB}$ cache. Raspberry Pi is neither a processor nor a controller, it is an embedded computer. 


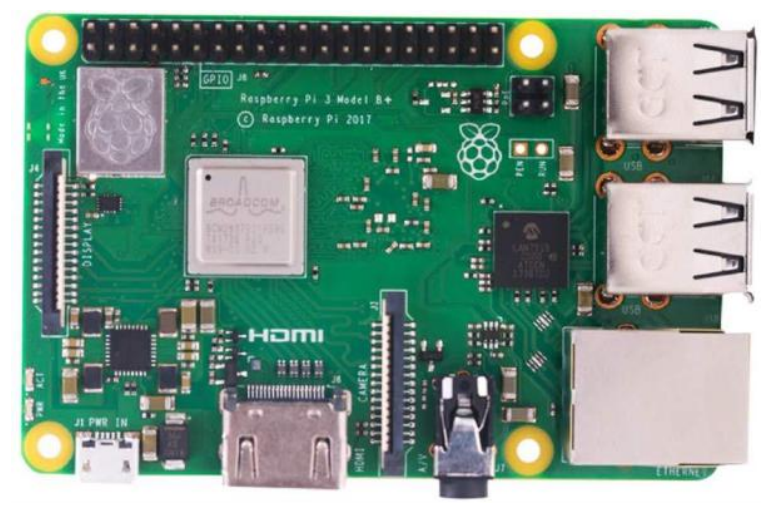

Figure 2 Raspberry Pi 3 (Model B+)

The distance is measured by sending ultrasonic waves in the ultrasonic sensor which is shown in fig.3. This wave hit the target and reflected back to measure distance. Ultrasonic sensor measures the distance by time between emitted and reflected wave. Eq.1 shows the formula to calculate the distance.

$$
\operatorname{Dis} \tan c e(D)=\frac{1}{2} t c
$$

Where $D$ is the distance, $t$ is the time between the emission and reception, and $c$ is the sonic speed. The value is multiplied by $\frac{1}{2}$ because $t$ is the distance time for go-and-return.

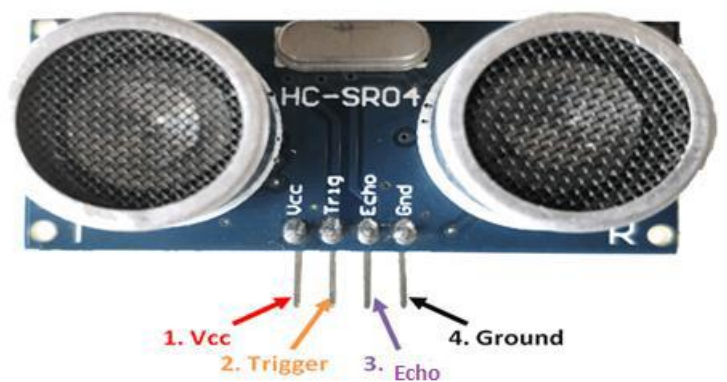

Figure 3 Ultrasonic Sensor

A Webcam is a video camera that feeds or streams an image or video to a computer network, such as the internet, in real time, to or from a computer. Usually, webcams are tiny cameras that sit on a desk, connect to a user's computer, or are built into the hardware. During a video chat session between two or more participants, with conversations like live audio and video, webcams may be used. The flow chart of object detection is shown in Fig.4. 


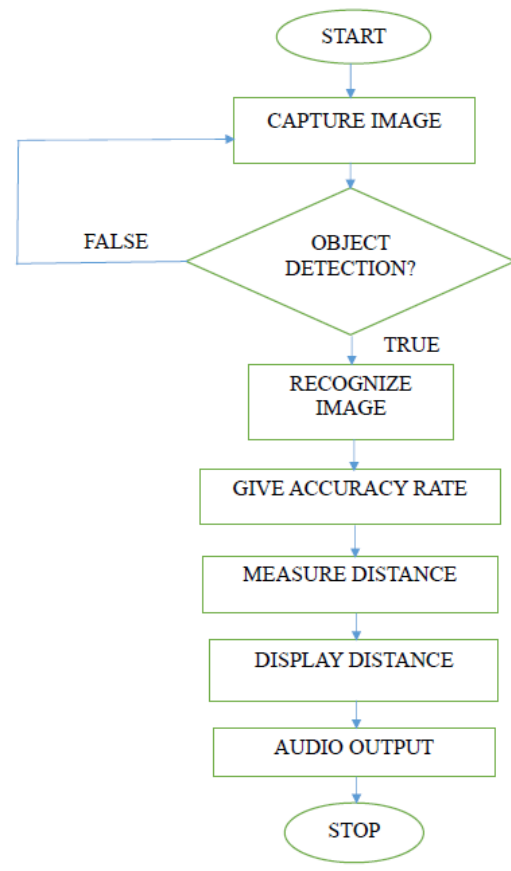

Figure 4 Flow Chart of Object Detection

\section{Results and Discussion}

This proposed work is an attempt to help the visually impaired people to prevent accidents and to detect people and the objects around them using the machine learning algorithm. The system or the kit uses a web camera which is interfaced with the Raspberry $\mathrm{Pi}$, the processor control. The processor captures images from the real time video camera which is recognized, compared and then detected. With the help of this machine learning certain data sets are trained using the Tensor Flow Library files in order to detect the objects or the faces in front of the visually impaired people. Python IDE 3.0 is the programming language used in here to train and test the images. The kit also consists an Ultrasonic sensor which then detects the distance between the object and the person, which is then converted into speech with the help of Pico text to speech converter and finally the output is delivered through the headset connected to the processor. Input voltage of $5 \mathrm{~V}$ and input current of $2 \mathrm{~A}$ is supplied via the Micro USB port. Raspberry $\mathrm{Pi}$ is used as the processor which processes the image accordingly and helps to detect the image. A web camera is connected to the Raspberry Pi to capture the live image and an ultrasonic sensor soldered in a PCB to detect the distance between the object detected and the prototype. Headphones are connected to the Raspberry Pi to hear out the audio converted from text to speech. Fig.5 shows the complete prototype for Bionic Eye system.

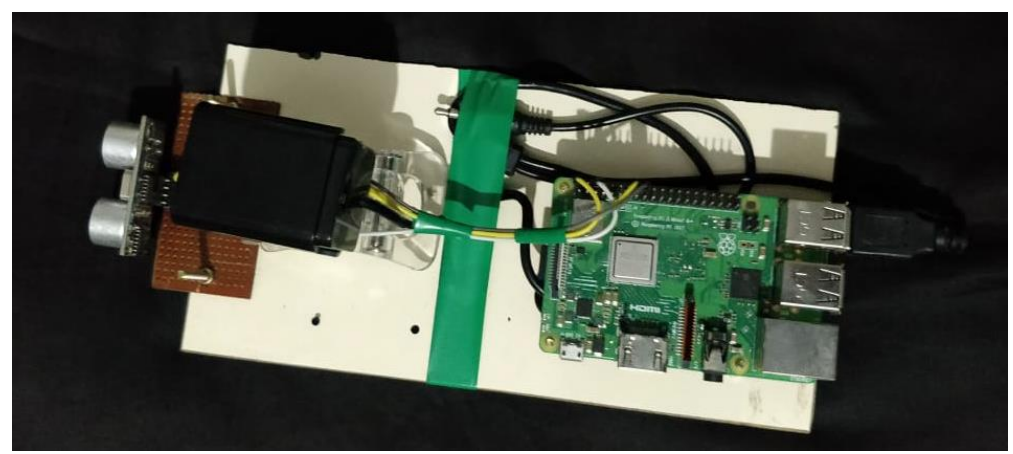

Figure 5 Bionic Model

At first Anaconda virtual environment is installed to manage the files of python along with its requiring libraries as shown in fig.6. The environmental variable path is configured and the requiring packages for Tensor flow are also installed. The datasets are collected and are labeled using the labeling Tool. 


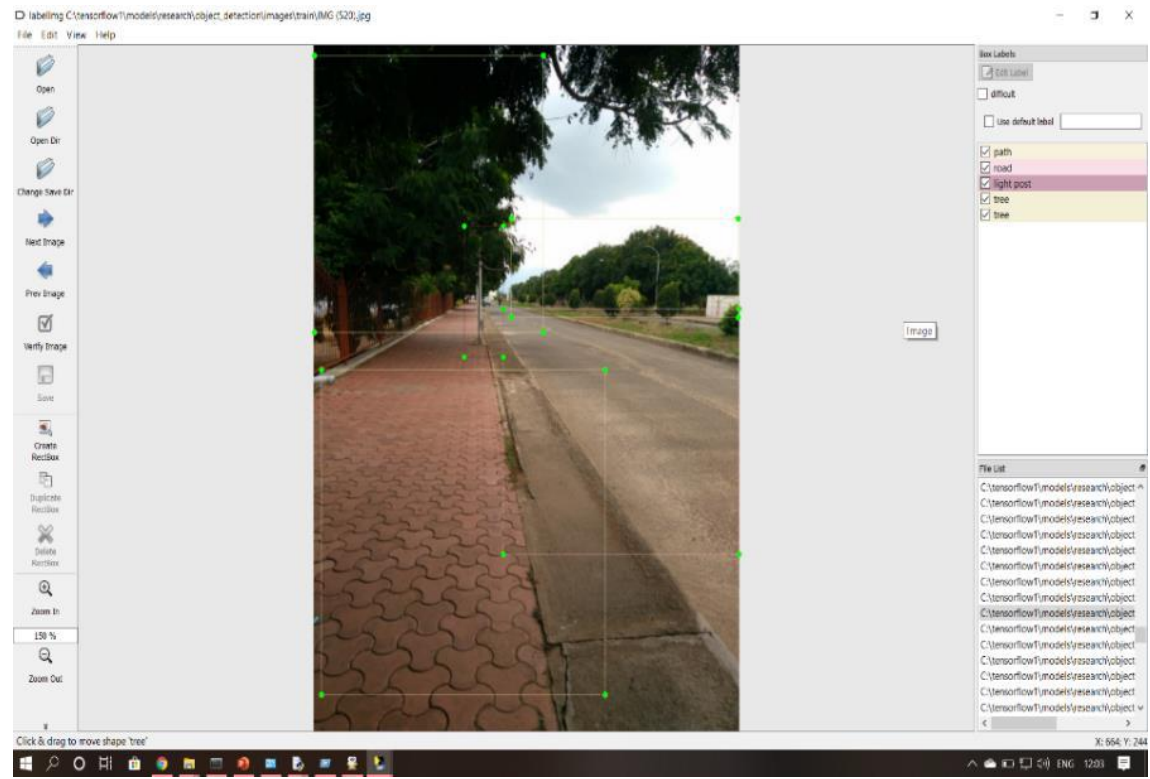

Figure 6 Screenshot of labeling the image using Labeling Tool

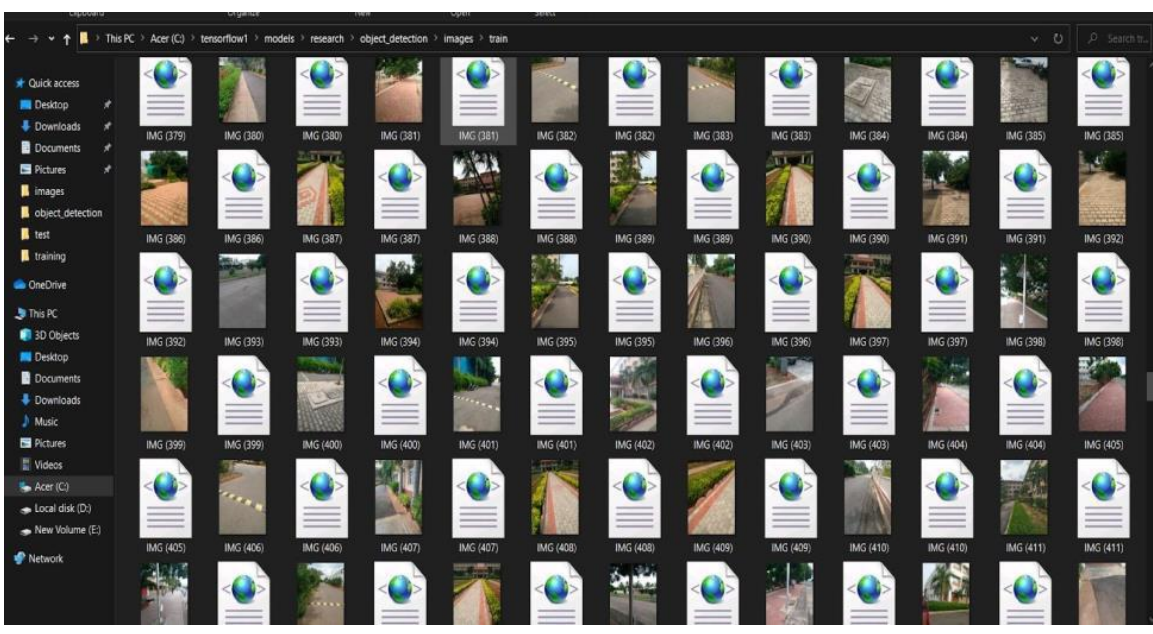

Figure 7 Screenshot of the train and test directory

The images labeled are to be trained which are to be converted from xml to csv.py files using the code. The images shown in fig 7 explain this process. These rained images along with tensor flow library files are shifted to the Raspberry pi software. The images are labeled to be predicted using the code so that the object can be detected in real time. As executing, the objects in the screen captured in frames per second through the web camera are detected according to the datasets given, in which some are Pets, bikes, cars and tress which tends to be some of the common objects available in the roads as it is necessary to identify them for the visually aided to be safe. The output is shown in fig.8, where the object is being identified with the help of its given features matching the object. 


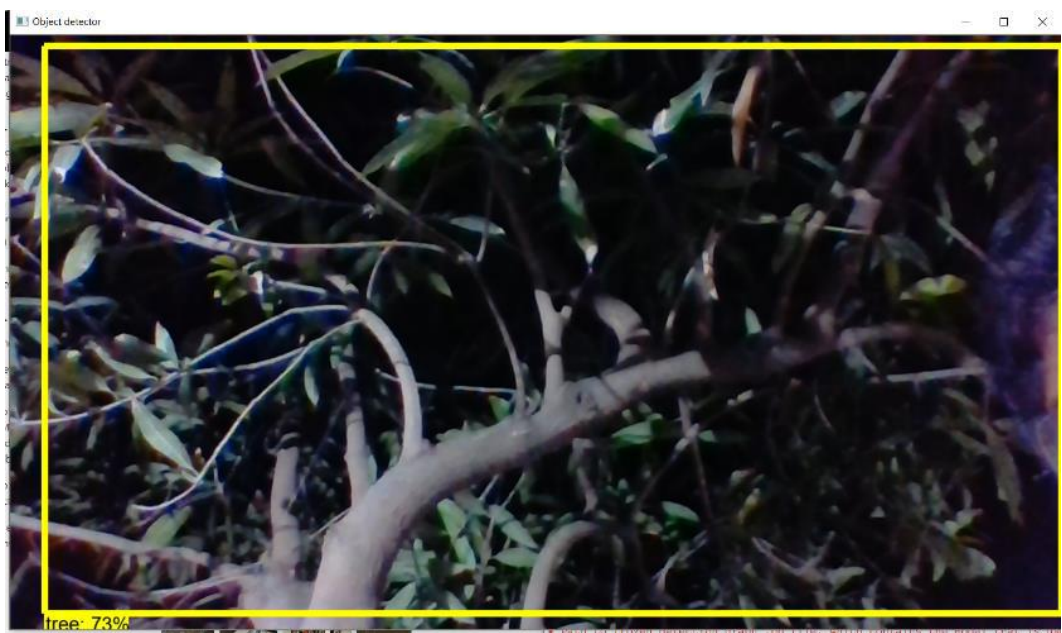

Figure 8 Screenshot of the Object (Tree) being detected by the model

After the object being identified as shown in fig.8, the distance of the object from the prototype is measured with the help of the Ultrasonic sensor where the ultrasonic rays are delivered from the sensor which hits the object in front and is received back by the sensor which is used to identify the distance. The distance measured is in the unit centimeter. This distance is then heard by the user using the text to speech convertor which helps them to notify them about the obstacle which is in the front. This paper presents the conversion of live stream into objects which are detected for the blind people.

\section{Conclusion}

In general, this research helps the visual aided person to identify the objects and the distance of that particular object in front of them. Here, using machine learning the datasets of frequently available insight objects was instructed to help that in need, to avoid accidents. In order to train those Machine learning models, the Tensor flow Lite Machine learning library was used which is an end-to-end open source platform. By processing the live video captured by the camera, the objects were detected efficiently by comparing the features of the object. Finally, after detecting the object, the distance of that object was measured using sensor which converts text to speech. This speech alerts them about the obstacles and prevents accidents. Thus this prototype will guide the visually aided people with handy support and will ensure them to have a safe surrounding by helping them to avoid unnecessary collisions

\section{References}

A. H. Pech, P. M. Nauth and R. Michalik, 2019 "A new Approach for Pedestrian Detection in Vehicles by Ultrasonic Signal Analysis," IEEE EUROCON 2019 -18th International Conference on Smart Technologies, Novi Sad, Serbia, pp. 1-5.

C. T. Patel, V. J. Mistry, L. S. Desai and Y. K. Meghrajani, 2018, "Multisensor -Based Object Detection in Indoor Environment for Visually Impaired People," Second International Conference on Intelligent Computing and Control Systems (ICICCS), Madurai, India, pp. 1-4.

B. Deepthi Jain, S. M. Thakur and K. V. Suresh, 2018, "Visual Assistance for Blind Using Image Processing," International Conference on Communication and Signal Processing (ICCSP), Chennai, pp. 0499- 0503.

Diwakar Srinath A, Praveen Ram A.R, Siva R, Kalaiselvi V.K.G and Ajitha G, 2017, "HOT GLASS - human face, object and textual recognition for visually challenged," 2nd International Conference on Computing and Communications Technologies (ICCCT), Chennai, pp. 111-116.

S. S. Walam, S. P. Teli, B. S. Thakur, R. R. Nevarekar and S. M. Patil, 2018, "Object Detection and seperation Using Raspberry PI," Second International Conference on Inventive Communication and Computational Technologies (ICICCT), Coimbatore, pp. 214-217.

S. N. Kulkarni and S. Kumar Singh, 2018, "Object Sorting Automated System using Raspberry Pi," 3rd International Conference on Communication and Electronics Systems (ICCES), Coimbatore, India, pp. 217220.

R. Kumar and S. Meher, 2015, "A Novel method for visually impaired using object recognition," International Conference on Communications and Signal Processing (ICCSP), Melmaruvathur, pp. 0772-0776.

V. K. Bhanse and M. D. Jaybhaye, 2018, "Face Detection and Tracking Using Image Processing on Raspberry $\mathrm{Pi}$," International Conference on Inventive Research in Computing Applications (ICIRCA), Coimbatore, pp. 1099-1103. 
P.Velrajkumar, P.Ramesh, C.Senthilpari, T.Bhuvaneswari, V.Chitra, 2020, "Development of Autonomous Robot for Tunnel Mapping using Raspberry-Pi Processor", International Journal of Scientific and Technology Research, 9 (3), pp. $191-194$.

Sathya Priya, P.Velrajkumar, R.Thenmozhi, J.Joyce Jacob, C.Sethilpari, 2020, "Development of Six Axes Robotic Arm Manipulator using Android Application”, Solid State Technology, 63 (6), pp. 8082 - 8089.

P.Velrajkumar, C.Senthilpari, P.Ramesh, G.Ramanamurthy, D.Kodandapani, 2019, "Development of Smart Number Writing Robotic Arm using Stochastic Gradient Decent Algorithm", International Journal of Innovative Technology and Exploring Engineering, 8 (10), pp. 542 - 547.

P.Velrajkumar, G.Ramanamurthy, J.Emerson Raja, Md.Jakir Hossen, Lo Kok Jong, 2017, "Controlling and Tracking of Mobile Robot in Real-Time using Android Platform", Journal of Engineering and Applied Sciences, 12 (4), pp. $929-932$. 